FERMINO MH; TREVISAN M; BUSNELLO AC. 2015. Cascas de tungue e de noz pecan como alternativa de substrato para horticultura. Horticultura Brasileira 33: 459-464. http://dx.doi.org/10.1590/S0102-053620150000400009

\title{
Cascas de tungue e de noz pecan como alternativa de substrato para horticultura
}

\author{
Maria H Fermino; Miriam Trevisan; Ângela C Busnello \\ Fundação Estadual de Pesquisa Agropecuária do Rio Grande do Sul (FEPAGRO), Porto Alegre-RS, Brasil; maria-fermino@fepagro. \\ rs.gov.br
}

\section{RESUMO}

Um substrato geralmente é composto por diferentes componentes como turfa, perlita, vermiculita e cascas de árvores, como pinus e eucalipto, além de diversos outros resíduos. O uso de resíduos como substrato para plantas pode propiciar a obtenção de materiais alternativos, com estrutura estável, de fácil obtenção, constante disponibilidade e com baixo custo. Para tanto, buscou-se neste trabalho descrever dois resíduos agroindustriais com potencial de uso como substrato para plantas: cascas de noz pecan e de tungue trituradas e decompostas, de forma aeróbica e anaeróbica. Os materiais foram analisados física e quimicamente e misturados à turfa marrom in natura em seis proporções de misturas [100:0; 80:20; $60: 40 ; 40: 60 ; 20: 80 ; 0: 100(\mathrm{v}: \mathrm{v})]$. Tanto os materiais puros quanto as misturas foram analisados quanto a densidade, porosidade, espaço de aeração e disponibilidade de água, valor de $\mathrm{pH}$ e condutividade elétrica. Os resíduos, cascas de noz pecan e de tungue, triturados, apresentaram características físicas e químicas adequadas para a utilização como substrato, puros e nas misturas com turfa.

Palavras-chave: Aleurites sp., Carya illinoensis, propriedades físicas e químicas.

\begin{abstract}
Husks tung and pecan nut as alternative substrate for horticulture

A substrate is generally composed of different components such as peat, perlite, vermiculite, bark, as pine and eucalyptus, and various other wastes. The employment of waste as substrate to cultivate plants may provide alternative materials with stable structure, easy to obtain, constant availability and low cost. Therefore, we sought to describe two agro-industrial waste with potential use as a substrate for plants: crushed shells of pecan and tung decomposed aerobically and anaerobically. Materials were analyzed chemically and physical and mixed with brown peat in natura in six proportions of mixtures $(100: 0 ; 80: 20 ; 60: 40 ; 40: 60 ; 20: 80 ; 0: 100)$. The pure material and the mixtures were analyzed for density, porosity, aeration and water availability, $\mathrm{pH}$ value and electrical conductivity. The waste, walnut pecan and tung husks, crushed, presented physical and chemical characteristics suitable for use as substrate, in mixtures with peat and in isolated form.
\end{abstract}

Keywords: Aleurites sp., Carya illinoensis, physical and chemical properties.

(Recebido para publicação em 12 de fevereiro de 2014; aceito em 14 de maio de 2015)

(Received on February 12, 2014; accepted on May 14, 2015)

$\mathrm{O}$ investimentos financeiros e tecnológicos na horticultura brasileira passaram a alavancar produções em grande escala, resultado da percepção do grande mercado consumidor que o país apresenta. Dentre os insumos agrícolas, a escolha certa do substrato determina o melhor aproveitamento de outros fatores, como água e adubos, determinando uma melhor qualidade do produto final.

Levantamento feito pela ABISOLO (Associação Brasileira das Indústrias de Substratos, Fertilizantes Orgânicos e Condicionadores de Solo), dentre as empresas associadas, mostra que em 2009 as empresas do setor comercializaram aproximadamente $54 \mathrm{mil} \mathrm{m}^{3}$ de substrato. No entanto, estimam que o mercado brasileiro seja de $900 \mathrm{mil} \mathrm{m}^{3}$ de substrato (ABISOLO, 2009).

No cultivo em recipientes, independente do substrato utilizado, observa-se uma limitação de espaço para a expansão das raízes. Esta limitação exige que o substrato seja capaz de manter o volume de água facilmente disponível às plantas sem, no entanto, comprometer a concentração de oxigênio no meio (Bunt, 1961). Assim, na hora de selecionar ingredientes para compor um substrato, torna-se importante a escolha de materiais com adequadas características físicas para um dado recipiente, um determinado manejo e para aquela espécie em questão.

Devido às excelentes qualidades da turfa, como alto teor de matéria orgânica, baixo valor de $\mathrm{pH}$, baixa disponibilidade de nutrientes, alto poder tampão, alta capacidade de retenção de água e boa aeração, esta tornou-se o principal componente para elaboração de substratos hortícolas (Burés, 1997), sendo utilizada como padrão de comparação para estudo de novos materiais.

Observa-se a partir da década de 80 , em países europeus, a busca de materiais substitutos à turfa, principal componente internacional para a elaboração de substratos, devido à exaustão de reservas naturais e/ou elevação do preço para 
importação. No entanto, atualmente, na Alemanha (Schmilewski, 2009), na França (Rivière et al., 2008) e no reino Unido (Holmes, 2009), os substratos ainda comercializados são compostos principalmente por turfa.

Existem referências em literatura ao uso de diversos materiais componentes de substratos, como estercos, cascas de árvores, fibras naturais, lixo urbano, poliuretanos, rochas naturais, entre outros (Worral, 1978; Verdonck, 1984; Schmilewscki, 1991; Lamanna et al., 1991; Cole \& Newell, 1996; Baumgarten, 2008).

$\mathrm{Na}$ última década, na Europa Ocidental e nos Estados Unidos, percebe-se um incremento no uso de cascas de Pinus compostadas. Na Austrália, este é o principal componente de substratos (Carlile, 2008). Da mesma forma, a Nova Zelândia consome uma mistura típica de substrato, que utiliza 40\% de turfa, $40 \%$ de cascas de Pinus compostadas e $20 \%$ de pedra-pome (Smith, 2008).

A indústria brasileira utiliza para compor seus substratos, principalmente, casca de Pinus, turfa, vermiculita e perlita (Müller, 2000). Outros materiais seriam as cascas de eucalipto e a fibra de coco. Na busca de componentes com a melhor qualidade, a indústria nacional os adquire de diversas partes do país ou de outros países: fibra de coco do Pará (Amafibra, 2015); turfa marrom de Santa Catarina (Florestal, 2015); a perlita de origem vulcânica pode vir da Argentina (Schumacher, 2015); a vermiculita vem da África do Sul e dos Estados Unidos (Barbosa, 2015); turfa de esfagno do Canadá (Veja \& Flora, 2015).

No RS, produtores vêm utilizando cascas de noz pecan para compor substratos para o cultivo de orquídeas. A empresa "Pecuária Link" de Cachoeira do Sul-RS possuía, em 2007, 200 mil árvores de noz pecan e produzia 150 mil kg/ano de resíduo ao custo de $\mathrm{R} \$$ $0,50 / \mathrm{kg}$. Já a casca de tungue, um dos resíduos da indústria de óleo de tungue ("tung oil" ou "wood oil"), composto pela casca do fruto e testa de sementes (Gruszynski, 2002), é usada como condicionador de solo no cultivo de rosas e de crisântemos de corte. A disponibilidade média do resíduo no RS é da ordem de $3.000 \mathrm{~m}^{3}$ ao ano (Gruszynski, 2002).
O uso de resíduos como substrato para plantas pode propiciar a obtenção de materiais alternativos, com estrutura estável, de fácil obtenção, constante disponibilidade e com baixo custo. Para tanto, buscou-se caracterizar a partir das propriedades físicas (densidade, porosidade, espaço de aeração e disponibilidade de água) e químicas (valor de $\mathrm{pH}$ e condutividade elétrica) os resíduos, cascas de noz pecan e de tungue, em diferentes combinações com turfa marrom, para o uso como substratos para plantas.

\section{MATERIAL E MÉTODOS}

O tratamento dos resíduos teve início em novembro de 2005, nas dependências do setor de Floricultura da Faculdade de Agronomia da UFRGS. As análises físicas e químicas ocorreram no Laboratório de Análises de Substrato da Fepagro, em Porto Alegre.

As cascas de noz pecan (Carya illinoensis) foram fornecidas pela empresa Orquidarium, localizada no município de Guaíba-RS. O resíduo é originário da "Pecuária Link" de Cachoeira do Sul-RS. As cascas, após trituradas em moinho tipo martelo foram colocadas em um recipiente com água para a eliminação do tanino numa proporção de 1 resíduo: 2 água (volume:volume). Semanalmente, trocou-se a água e uma amostra foi coletada para medir o valor da condutividade elétrica e do pH. Quando da estabilização, após sete semanas, o material foi seco ao ar livre.

As cascas de tungue (Aleurites sp.) provenientes do município de Veranópolis-RS, após a moagem foram utilizadas de duas formas. Uma parcela foi posta a decompor ao ar livre, sendo remexida semanalmente, até estabilização, que ocorreu em 11 semanas [tungue aeróbico (TAERO)]. Outra parcela foi decomposta em água numa proporção de 1 resíduo: 3 água (volume:volume) [tungue anaeróbico (TANA)]. Semanalmente, trocou-se a água e uma amostra foi coletada para medir o valor da condutividade elétrica e do pH. Após a estabilização, que ocorreu, em sete semanas, procedeu-se a secagem ao ar livre.
Para compor as misturas foi usada turfa marrom in natura $(\mathrm{T})$, proveniente da empresa Florestal SA de Araranguá-SC. As misturas NP:T, TAERO:T e TANA:T foram feitas nas proporções de 100:0; 80:20; 60:40; 40:60; 20:80; 0:100 (relação v:v) e submetidas às analises químicas e físicas, em número de três repetições.

Para a determinação da densidade seca (DS), valor de $\mathrm{pH}(\mathrm{pH})$ e condutividade elétrica (CE) foram empregados os métodos descritos na Instrução Normativa $N^{\circ} 17$ do MAPA (Ministério da Agricultura, Pecuária e Abastecimento) (Brasil, 2007).

A determinação da porosidade total (PT), do espaço de aeração (EA), água facilmente disponível (AFD), água tamponante (AT) e água remanescente a 50 (AR50) e $100 \mathrm{~cm}$ (AR100) foi realizada através de curvas de retenção de água nas tensões de $0,10,50$, e 100 $\mathrm{cm}$ de altura de coluna de água, correspondendo às tensões de $0,10,50$ e 100 hPa, para TAERO e TANA, e de 0,10 e $50 \mathrm{hPa}$ para NP, conforme De Boodt \& Verdonck (1972).

Todos as análises das características físicas (DS, PT, EA, AFD, AT e AR) e químicas ( $\mathrm{pH}$ e $\mathrm{CE}$ ) foram realizadas em delineamento completamente casualizado e em número de três repetições. Os resultados foram submetidos à análise de variância e de regressão, utilizando o Programa Sigma STAT versão 2.0.

\section{RESULTADOS E DISCUSSÃO}

Após a trituração as cascas de noz pecan apresentaram 11\% das partículas com granulometria acima de 4,75 $\mathrm{mm}$, enquanto as de tungue apenas 3,5\%; $11 \%$ das partículas de noz pecan estavam entre 3,35 e $4,75 \mathrm{~mm}$, enquanto as de tungue $18 \%$; o maior volume das partículas de noz pecan, $47 \%$, ficaram na malha entre 2,00 e $3,35 \mathrm{~mm}$, enquanto as de tungue $34 \%$; e finalmente, $31 \%$ das partículas de noz pecan apresentaram granulometria menor do que 2,00 mm, enquanto que as de tungue foram $44 \%$. Embora com diferenças entre as classes, tanto as cascas de noz pecan como as de tungue apresentaram 78\% de partículas com granulometria menor do que 
3,35 mm e, consequentemente, $22 \%$ de partículas maiores. As diferenças entre densidade, porosidade e disponibilidade de água apresentadas pelos materiais e discutidas ao longo do trabalho podem ser explicadas não necessariamente pelo

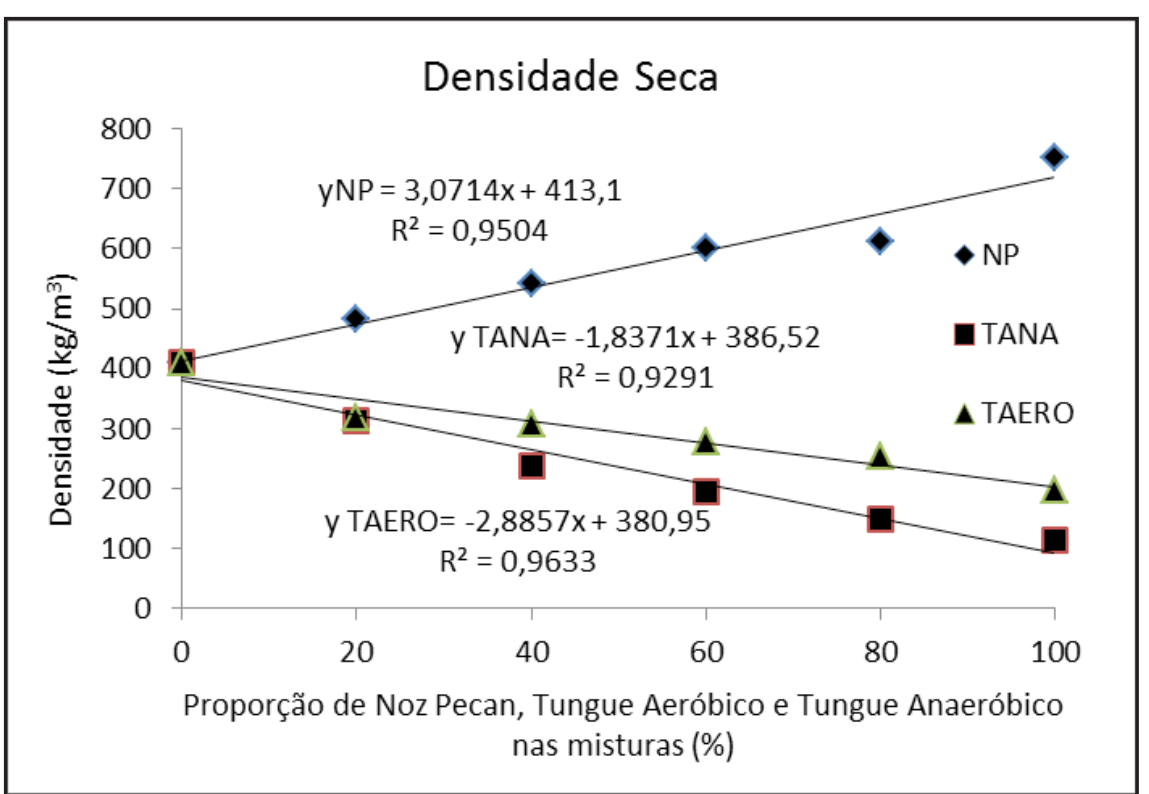

Figura 1. Análise de regressão da densidade seca $\left(\mathrm{kg} / \mathrm{m}^{3}\right)$ das misturas de turfa marrom in natura com noz pecan (NP), tungue anaeróbico (TANA) e tungue aeróbico (TAERO), em percentual de volume (média de três amostras) \{regression analysis of dry density (kg/ $\mathrm{m}^{3}$ ) mixtures of brown peat in natura with pecan walnut husks (NP), anaerobic (TANA) and aerobic tung husks (TAERO), in percentage of volume (three samples average) $\}$. Porto Alegre, FEPAGRO, 2007.

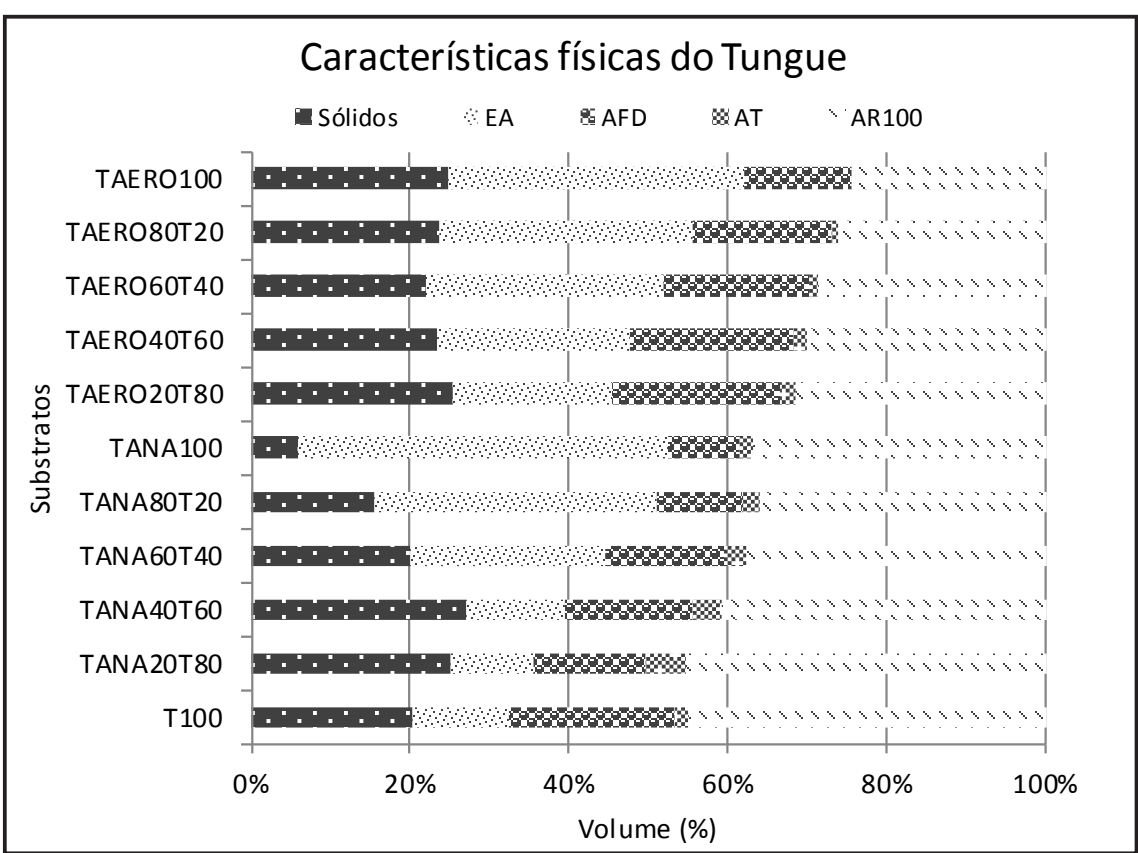

Figura 2. Características físicas das misturas de turfa marrom in natura (T) com tungue anaeróbico (TANA) e com tungue aeróbico (TAERO), em volume (\%) (média de três amostras): sólidos, espaço de aeração (EA), água facilmente disponível (AFD), água tamponante (AT) e água remanescente a $100 \mathrm{~cm}$ (AR100) \{physical characteristics of mixtures of brown peat in natura (T) with anaerobic (TANA) and aerobic tung husks (T), by volume (\%) (average of three samples): solid, air space (EA), easily available water (AFD), tamponant water (AT) and remaining water at $100 \mathrm{~cm}$ (AR100) (average of three samples) $\}$. Porto Alegre, FEPAGRO, 2007. "tamanho" das partículas, mas segundo Burés (1997), por outras características como rugosidade e estrutura interna. Assim, as múltiplas combinações possíveis de partículas distintas e sua disposição podem explicar a complexidade da caracterização física.

Os materiais à base de tungue, TANA e TAERO, puros, apresentaram baixos valores de densidade seca (DS) (113 e $197 \mathrm{~kg} / \mathrm{m}^{3}$, respectivamente), valores estes inferiores à turfa $\left(410 \mathrm{~kg} / \mathrm{m}^{3}\right)$. Consequentemente, a adição de TANA e TAERO à turfa, em todas as proporções propostas, reduziu a densidade original da turfa (Figura 1).

De maneira geral, segundo Kämpf (2005), para propagação em células e bandejas, um substrato deve apresentar densidade entre 100 e $300 \mathrm{~kg} / \mathrm{m}^{3}$, para vasos de até $15 \mathrm{~cm}$ de altura, o mesmo deve ter entre 200 e $400 \mathrm{~kg} / \mathrm{m}^{3}$ e para vasos de 20 a $30 \mathrm{~cm}$ de altura, um substrato deve ter entre 300 a $500 \mathrm{~kg} /$ $\mathrm{m}^{3}$. Substratos com densidade superior a $500 \mathrm{~kg} / \mathrm{m}^{3}$ só devem ser utilizados para vasos maiores. Considerando os valores sugeridos por Kämpf, as misturas e os materiais puros propostos, permitem uma ampla gama de utilização, seja em bandejas ou pequenos vasos.

TANA apresentou 94\% de porosidade total (PT), enquanto a turfa apresentou $80 \%$. À exceção das misturas TANA 20:80 (75\%), TANA 40:60 (73\%) todos os valores de porosidade apresentados encontram-se próximos ou acima do valor sugerido (85\%) para esta variável (Figura 2), segundo De Boodt \& Verdonck (1972). O alto valor da porosidade de TANA na forma pura é característica pouco verificada na literatura, assemelhando-se ao valor da fibra de coco (95\%) (Amafibra, 2013).

TANA também apresentou expressivo valor de espaço de aeração (EA) (46\%) (Figura 2), característica esta também pouco verificada na literatura. No entanto, à medida que diminuiu o percentual de TANA nas misturas, os valores do EA diminuíram, até estabilizarem-se em TANA 20:80 (11\%), TANA 40:60 (12\%), com valores semelhantes à turfa $(12 \%)$. Estes valores são considerados baixos quando comparados à faixa sugerida por De Boodt \& Verdonck (1972), entre 20 e 40\%. No entanto, a 
adequação ou não do espaço de aeração é dependente das exigências da espécie em questão e do sistema de cultivo. O manejo de um substrato com baixo espaço de aeração exige, dependendo da espécie, irrigações mais frequentes (Miner, 1994).
Contrariamente, o valor da água facilmente disponível (AFD) de TANA é baixo (10\%) praticamente metade do verificado na turfa (21\%) (Figura 2), cujo valor está dentro do sugerido por De Boodt \& Verdonck (1972), entre 20 e $30 \%$. Em todas as proporções, a mistura

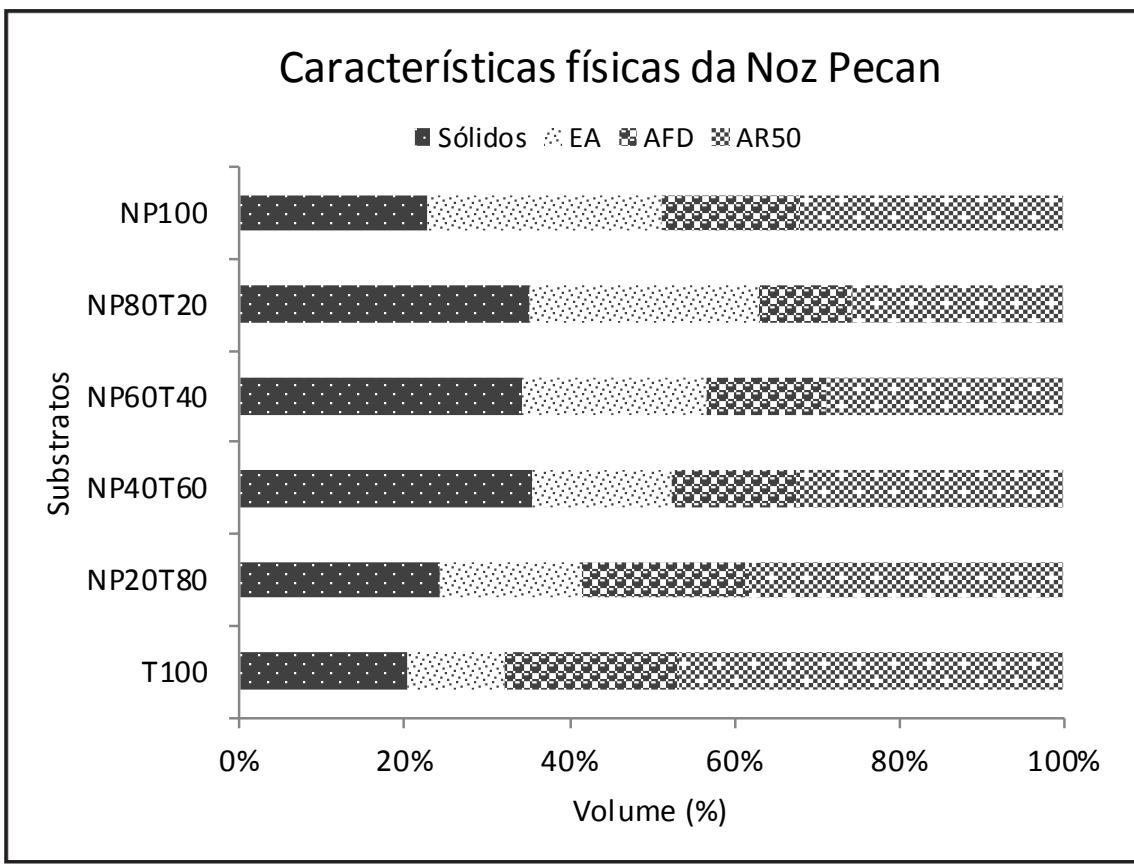

Figura 3. Características físicas das misturas de turfa marrom in natura (T) com noz pecan (NP), em volume (\%), média de três amostras: sólidos, espaço de aeração (AE), água facilmente disponível (AFD) e água remanescente a $50 \mathrm{~cm}$ (AR50) \{physical characteristics of mixtures of brown peat in natura (T) with pecan walnut (NP), by volume (\%), three average samples: solids, air space (AE), easily available water (AFD) and the remaining water at 50 cm (AR 50)\}. Porto Alegre, FEPAGRO, 2007.

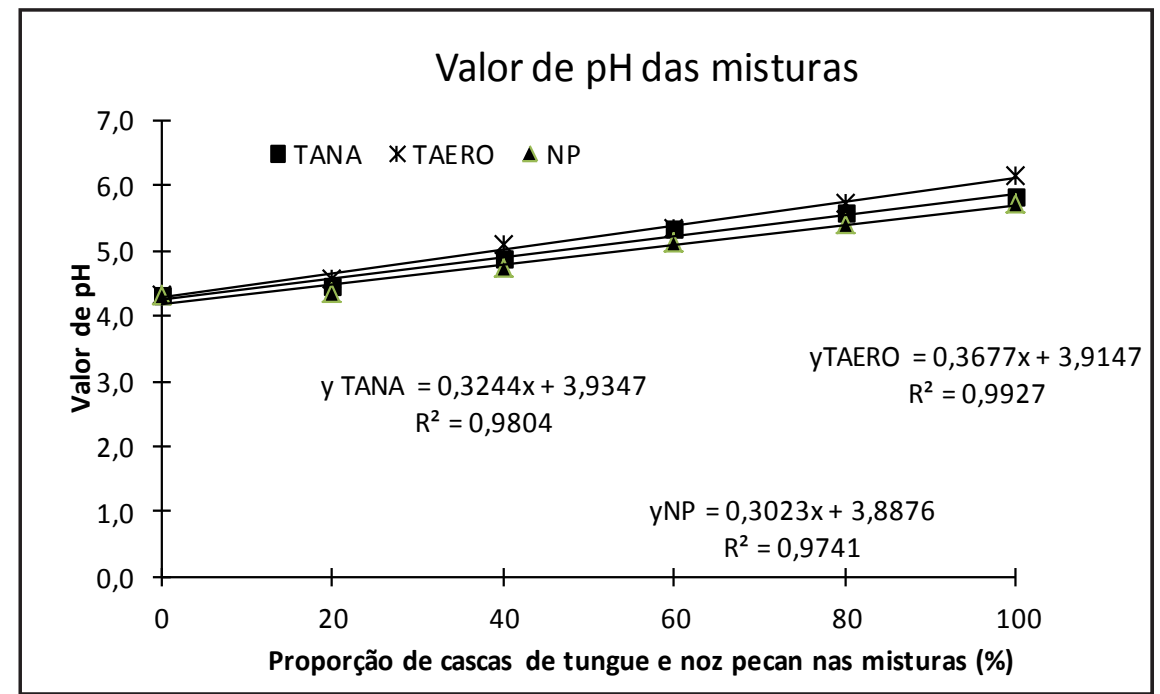

Figura 4. Análise de regressão dos valores de $\mathrm{pH}$ das misturas de turfa marrom in natura com tungue anaeróbico (TANA), tungue aeróbico (TAERO) e noz pecan (NP), em percentual de volume (média de três amostras) \{regression analysis of $\mathrm{pH}$ values of mixtures of brown peat in natura with anaerobic (TANA), aerobic tung husk (TAERO) and walnut pecan (NP), in percentage of the volume (average of three samples) \}. Porto Alegre, FEPAGRO, 2007.

de TANA à turfa reduziu a disponibilidade de água em comparação ao valor original da turfa (Figura 2).

O material TAERO apresentou valor de porosidade total $(75 \%)$ inferior à turfa $(80 \%)$ e, em todas as misturas, a adição de TAERO reduziu o valor da PT original da turfa (Figura 2). TAERO também apresentou valor para água facilmente disponível (14\%) inferior à turfa (21\%) e em todas as misturas, a adição de TAERO reduziu o valor da AFD original da turfa (Figura 2).

No entanto, de forma semelhante a TANA, TAERO apresentou um alto valor de espaço de aeração (37\%), o qual diminui proporcionalmente com o aumento do teor de turfa na mistura (Figura 2). Embora TAERO apresente uma redução no valor do EA em relação à TANA (37 e 46\%, respectivamente) ainda assim, estes são valores bastante expressivos e foram pouco modificados pelo tratamento a que foi submetido o material, decomposição aeróbica ou anaeróbica.

Segundo Miner (1994), para substratos com partículas de dimensões compreendidas entre um e $10 \mathrm{~mm}$, tanto a porosidade como a quantidade de água retida variam pouco com o tamanho da partícula. Partículas de tamanho menor de um mm causam um brusco decréscimo da porosidade e aumento da retenção de água. Handreck \& Black (1999) demonstram que o aumento da proporção de partículas com tamanho entre 0,1 e $0,25 \mathrm{~mm}$ e menores, em substratos à base de cascas de Pinus, aumentam o volume de água retida pelo substrato e reduzem a disponibilidade de ar.

A NP apresentou o mais alto valor de densidade seca $\left(752 \mathrm{~kg} / \mathrm{m}^{3}\right)$; consequentemente, todas as misturas propostas com este material elevaram a densidade original da turfa $\left(410 \mathrm{~kg} / \mathrm{m}^{-3}\right)$ (Figura 1). Embora, aparentemente, o valor de densidade da NP esteja acima do recomendado (Kämpf, 2005), exigências específicas da planta ou espécie ocorrem, como é o caso do cultivo de orquídeas, para o qual se busca substratos com alta densidade, de forma a manter os vasos em pé, e alta porosidade total aliada com alto espaço de aeração, para a manutenção da oxigenação das raízes. Assim, a NP além da alta densidade também apre- 


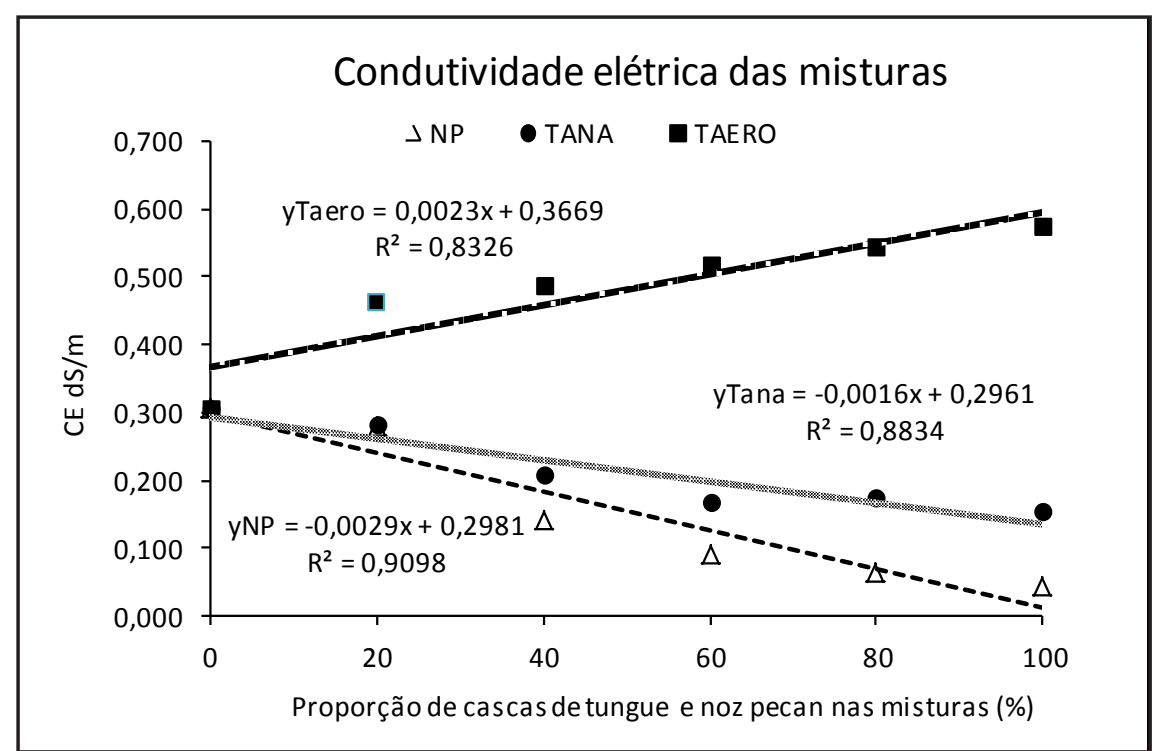

Figura 5. Análise de regressão dos valores de condutividade elétrica das misturas de turfa marrom in natura com tungue anaeróbico (TANA), tungue aeróbico (TAERO) e noz pecan (NP), em percentual de volume (média de três amostras) \{regression analysis of eletric conductivity of mixtures of brown peat in natura with anaerobic (TANA), aerobic tung husks (TAERO) and walnut pecan (NP), in percentage of the volume (average of three samples) $\}$. Porto Alegre, FEPAGRO, 2007.

senta valor de porosidade total de $77 \%$ e espaço de aeração de $29 \%$ (Figura 3). Nesta condição, a empresa Orquidarium fazia uso das cascas da noz pecan.

Todas as misturas de NP com turfa reduziram a PT em comparação aos valores originais dos materiais puros. O aumento da proporção de turfa nas misturas reduziu o espaço de aeração e aumentou a água facilmente disponível.

Todos os materiais, TANA, TAERO e NP, apresentaram valor de $\mathrm{pH}$ dentro da faixa recomendada e superior à turfa $(4,3)$ (TANA, 5,8, TAERO, 6,2 e NP, $5,7)$. No entanto, embora, a sua adição à turfa, independente da proporção utiliza$\mathrm{da}$, tenha elevado o valor de $\mathrm{pH}$ (Figura 4), ainda assim as misturas necessitam de correção para atingirem patamares recomendados. Embora algumas espécies como samambaias, bromélias, azaleias e coníferas desenvolvem-se em condições de pH entre 4,5 e 5,0 (Kämpf, 2005), de maneira geral, para materiais orgânicos recomenda-se valor de $\mathrm{pH}$ próximo a 5,8 , condição não atingida pelas misturas estudadas (Kämpf, 2005).

TANA e NP apresentam valores de CE $(0,156$ e $0,045 \mathrm{dS} / \mathrm{m})$ inferiores à turfa $(0,307 \mathrm{dS} / \mathrm{m})$ (Figura 5). Ambos, em mistura com a turfa, diminuíram,
Foliares, Orgânicos, Organominerais e Substratos para Plantas. 2009. Números Abisolo. Disponível em: <http://www. agricultura.gov.br/arq_editor/file/.../ ApresentacaoABISOLO.pdf $>$. Acessado em 02 de dezembro de 2011.

AMAFIBRA. Amafibra Fibras e Substratos Agrícolas da Amazônia Ltda. 2013. Disponível em $<$ http://www.amafibra.com.br/a-fibra-decoco/> Acessado em 24 de abril de 2015.

BARBOSA NS. 2015. Vermiculita: Aplicações. Disponível em: http://www.vermiculita.com. br/ Acessado em 24 de abril de 2015.

BAUMGARTEN A. 2008. Analytical methods for growing media - Challenges and perspectives. Acta Horticulturae 779: 97-104.

BUNT AC. 1961. Some physical properties of pot-plant composts and their affect on plant growth. Plant and Soil 12: 322-332.

BURÉS S. 1997. Materiales procedentes de yacimientos naturales, explotación minera y construcción. Turberas. In: Burés. Sustratos. Madrid: Ediciones Agrotécnicas. p.218 -258.

BRASIL. Ministério da Agricultura. 2007. Métodos para análise de substratos para plantas e condicionadores de solo. Instrução Normativa $N^{\circ} 17$, de 21 de maio de 2007. Publicado no Diário Oficial da União de 24/05/2007, Seção 1, Página 8. Disponível em <http://sistemasweb.agricultura.gov.br/ sislegis/action/detalhaAto.do? method $=$ cons ultarLegislacaoFederal $>$ Acessado em 21 de junho de 2013.

CARLILE WR. 2008. The use of composted materials in growing media. Acta Horticulturae 779: 321-328.

CAVINS TJ; WHIPKER BE; FONTENO WC; HARDEN B; MCCALL I; GIBSON JL. 2000. Monitoring and managing $\mathrm{pH}$ and EC using the Pour Thru Extraction Method. Horticulture Information Leaflet/NCSU, 590. Disponível em $<$ http://www2.ncsu.edu/unity/ lockers/project/hortsublab/> Acessado em 16 de março de 2015.

COLE JC; NEWELL L. 1996. Recycled paper influences container substrate physical properties, leachate mineral content, and growth of rose-of-sharon and Forsythia. HortTechnology 6: 79-83.

De BOODT M; VERDONCK O. 1972. The physical properties of the substrates in horticulture. Acta Horticulturae 26: 37-44.

FLORESTAL S.A. 2015. Produtos Turfa Fértil. Disponível em $<\mathrm{http}: / /$ www.florestalsa.com. br/index.php/empresa $>$ Acessado em 24 de abril de 2015.

GRUSZYNSKI C. 2002. Resíduo agroindustrial "casca de tungue" como componente de substrato para plantas. Porto Alegre: UFRGS. 99p. (Dissertação mestrado).

HANDRECK KA; BLACK ND. 1999. Growing Media for ornamental plants and turf. Sydney: Unsw Press. 448p.

HOLMES S. 2009. Growing media developments in the UK. Acta Horticulturae 819: 23-26.

KÄMPF AN. 2005. Produção Comercial de Plantas Ornamentais. Guaíba: Agropecuária. $254 \mathrm{p}$.

ABISOLO. Associação Brasileira das Indústrias de Condicionadores de Solo, Fertilizantes 
ANGELO G. 1991. Compost-based media as alternative to peat on ten pot ornamentals. Acta Horticulturae 294: 125-129.

MINER AM. 1994. Substratos: propriedades y caracterizacion. Madrid: Ediciones MundiPrens. 172p.

MÜLLER JJV. 2000. A demanda em substrato sob o ponto de vista dos usuários: na olericultura. In: KÄMPF AN; FERMINO MH (eds). Substrato para plantas: A base da produção vegetal em recipientes. Porto Alegre: Gênesis. p. 159-162.

RIVIERE LM, MOREL P; MICHEL JC, CHARPENTIER S. 2008. Growing media in French horticulture. Acta Horticulturae 779: 33-28.

SCHMILEWSKI GK. 1991. Quality control and use of composted organic wastes as components of growing media in the Federal Replubic of Germany. Acta Horticulturae 294: 89-98.

SCHMILEWSKI GK. 2009. Growing media constituents used in the EU. Acta Horticulturae 819: 33-40.

SCHUMACHER. Schumacher Insumos Perlita. 2015. Disponível em <http://www. schumacherinsumos.com.br/perlita.htm> Acessado em 24 de abril de 2015.
SMITH B. 2008. The growing media market in New Zealand. Acta Horticulturae 779: 179-184.

VEJA \& FLORA. Cultivo indoor. 2015. Disponível em $<$ https:/www.vegaeflora.com. br/todas-categorias/cultivo-indoor/substratos/ sunshine-turfa-canadense.html $>$ Acessado em 24 de abril de 2015.

VERDONCK O. 1984. Reviewing and evaluation of new materials used as substrates. Acta Horticulturae 150: 467-473.

WORRALL RJ. 1978. The use of composted wood waste as a peat substitute. Acta Horticulturae 82: 79-86. 